\title{
A Case of Peripartum Cardiomyopathy Affected by Left Ventricle Thrombus
}

\author{
Nobuhiro Takeuchi ${ }^{\mathrm{a}, \mathrm{e}}$, Kazumasa Emori ${ }^{\mathrm{a}}$, Junichi Soneda ${ }^{\mathrm{b}}$, Kaori Mohric, \\ Masanori Takada ${ }^{\mathrm{d}}$
}

\begin{abstract}
Peripartum cardiomyopathy (PPCM) mimicking dilated cardiomyopathy is defined as an idiopathic cardiomyopathy affecting perinatal or postnatal women with no cardiac history. It occurs in 1 in 20,000 births in Japan. A 25-year-old female without cardiology diseases presented to our hospital's emergency department with upper abdominal pain, exertional dyspnea, and leg edema. One month prior, she gave birth to her first child via vaginal delivery. At 36 weeks of gestation, she was diagnosed with pregnancy hypertension by an obstetrician. Ultrasonography and non-contrast computed tomography were performed and revealed an edematous gallbladder as well as dilated hepatic and inferior jugular veins. Chest X-ray revealed cardiomegaly, lung edema, and pleural effusion. She was diagnosed with congestive heart failure. Transthoracic echocardiogram (TTE) revealed a dilated left ventricle with a reduced ejection fraction (EF) of $17 \%$. Immediately after admission, diuretic agents, including furosemide and carperitide, were administered. Although her heart failure improved with sufficient diuretic treatment, thromboses measuring 16 $\times 8$ and $29 \times 11 \mathrm{~mm}$ in her left ventricle were revealed by TTE on day 10 . Heparin sodium and warfarin potassium were initiated, which reduced the thromboses. Her symptoms improved, although EF was not restored to normal function. She was discharged on day 40 and an outpatient clinic follow-up was scheduled. Long-term prognosis of PPCM is currently unknown, and meticulous follow-up of the patient is required. Here we present a case of PPCM that was affected by a left ventricular thrombus.
\end{abstract}

Keywords: Peripartum cardiomyopathy; Ventricle thrombus

Manuscript accepted for publication November 18, 2016

a Department of Internal Medicine, Kobe Tokushukai Hospital, 1-3-10 Kamitakamaru, Tarumi-ku, Kobe-shi, Hyogo 655-0017, Japan

bDepartment of Cardiovascular Surgery, Kobe Tokushukai Hospital, 1-3-10 Kamitakamaru, Tarumi-ku, Kobe-shi, Hyogo 655-0017, Japan

'Department of Laboratory Medicine, Kobe Tokushukai Hospital, 1-3-10 Kamitakamaru, Tarumi-ku, Kobe-shi, Hyogo 655-0017, Japan

${ }^{\mathrm{d}}$ Division of Cardiology, Department of Internal Medicine, Kawasaki Hospital, 3-3-1 Higashiyama-cho, Hyogo-ku, Kobe-shi, Hyogo 652-0042, Japan ${ }^{e}$ Corresponding Author: Nobuhiro Takeuchi, Department of Internal Medicine, Kobe Tokushukai Hospital, 1-3-10 Kamitakamaru, Tarumi-Ku, Kobeshi, Hyogo 655-0017, Japan. Email: nobuhiro.takeuchi@tokushukai.jp

doi: https://doi.org/10.14740/jmc2698w

\section{Introduction}

Peripartum cardiomyopathy (PPCM) is a cardiac condition which affects perinatal or postnatal women without any cardiology history of cardiac condition and mimics dilated cardiomyopathy. It is characterized by a reduced ejection fraction (EF). Although its frequency is not high, clinicians have to keep in mind this condition because of high mortality rate in its severe cases. PPCM, even with a normal heart beat, may sometimes develop ventricular thrombus due to reduced EF and hypercoagulable status during the perinatal period. We here present a case of PPCM that was affected by a left ventricular (LV) thrombus.

\section{Case Report}

A 25-year-old female without any medical history visited our emergency department with upper abdominal pain. She recollected suffering from a leg edema at 36 weeks of gestation due to pregnancy hypertension as diagnosed by an obstetrician. A month after she gave birth to her first child, she presented with worsening leg edema and exertional dyspnea. Simultaneously, she also had mild upper abdominal pain. She denied any use of tobacco or alcohol.

On admission, her blood pressure was $127 / 99 \mathrm{~mm} \mathrm{Hg}$, heart rate was 130 beats $/ \mathrm{min}$, body temperature was $36.4{ }^{\circ} \mathrm{C}$, and oxygen saturation was $97 \%$ on room air. Initial clinical examination revealed a weight of $55 \mathrm{~kg}$, and height of $165 \mathrm{~cm}$. Inspection of the palpebral conjunctiva revealed no evidence of anemia. Auscultation of the heart revealed diastolic and systolic heart murmurs (third and fourth heart sounds) in the cardiac apex. Auscultation of the lungs revealed no rale or other lung sounds. Physical examination of the patient revealed pitting edema in both legs but no cyanosis.

Blood chemistry analyses revealed the following: mildly elevated white blood cell counts $(9,220$ cells $/ \mu \mathrm{L})$, mild anemia (red blood cell count, $431 \times 10^{4}$ cells $/ \mu \mathrm{L}$; hemoglobin, $11.2 \mathrm{~g} /$ $\mathrm{dL})$, mild hypoproteinemia $(5.5 \mathrm{~g} / \mathrm{dL})$, mild hypoalbuminemia $(3.4 \mathrm{~g} / \mathrm{dL})$, mildly elevated lactate dehydrogenase $(245 \mathrm{IU} / \mathrm{L})$, slightly elevated serum creatinine levels $(0.84 \mathrm{mg} / \mathrm{dL})$, and remarkably elevated brain natriuretic peptide levels $(2,000 \mathrm{pg} /$ $\mathrm{mL}$ ). There was no evidence of coagulation dysfunction (prothrombin time, $71 \%$; activated partial thromboplastin time, 

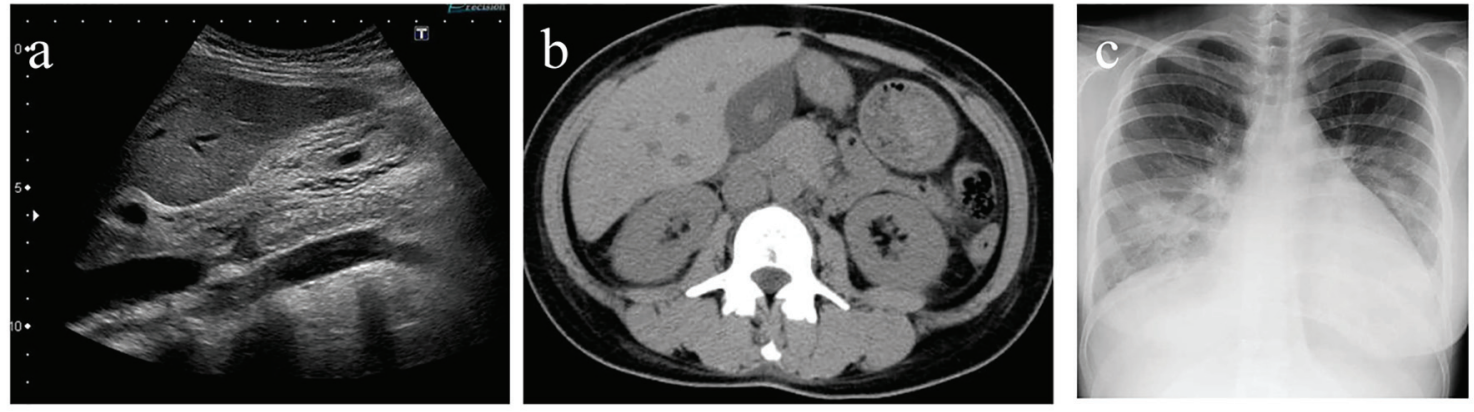

Figure 1. (a) Ultrasonography revealed an edematous gallbladder. (b) Non-contrast computed tomography revealed an edematous gallbladder, dilated hepatic and inferior jugular veins. (c) Chest X-ray revealed cardiomegaly with a cardio-thoracic ratio of $58 \%$, pulmonary congestion, and pleural effusion.

$26.2 \mathrm{~s})$.

Ultrasonography and non-contrast computed tomography were performed and revealed an edematous gall bladder and dilated hepatic and inferior jugular veins (Fig. 1a, b). Chest X-ray revealed cardiomegaly with a cardio-thoracic ratio of $58 \%$; moreover, there was evidence of pulmonary congestion and pleural effusion (Fig. 1c). Electrocardiogram revealed a normal sinus rhythm, heart rate of 130 beats $/ \mathrm{min}$, poor R progression in leads $\mathrm{V} 1-\mathrm{V} 3$, and inverted $\mathrm{T}$ wave in leads V4-V6. Transthoracic echocardiogram (TTE) revealed a dilated left ventricle of $63 \mathrm{~mm}$ in diastolic phase and 58 $\mathrm{mm}$ in systolic phase with a reduced EF of $17 \%$ (Fig. 2a, b). Color Doppler revealed moderate mitral regurgitation. At this time, she was diagnosed with congestive heart failure resulting from dilated cardiomyopathy, which was consistent with PPCM.

Immediately after admission, diuretic agents, including furosemide and carperitide, were administered. These improved her heart failure with sufficient diuretic effects. On day $6, \mathrm{X}$-ray revealed disappearance of heart congestion and cardiomegaly (Fig. 3). However, thromboses of $16 \times 8$ and $29 \times 11 \mathrm{~mm}$ in her left ventricle were revealed via TTE on day 10 (Fig. 4a, b). Heparin sodium and warfarin potassium were initiated, reducing the thromboses. While her symptoms improved, EF was not restored to normal function. She was discharged on day 40 and an outpatient clinic follow-up was planned.

\section{Discussion}

PPCM is a relatively uncommon cardiology condition which affects perinatal or postnatal women without any cardiac condition. In a US survey conducted between 2004 and 2011, the incidence of PPCM was 1 in 968 births [1], more than the Japanese rate of 1 in 20,000 births [2]. The definition of PPCM includes the following criteria [3]: 1) onset of new congestive heart failure between 1 month before and up to 5 months after; 2) history of cardiac condition, 3) no other causes of heart failure; and 4) LVEF $<45-50 \%$, LV fractional shortening of $<$ $30 \%$, and LV end-diastolic dimension/body mass index of $>$ $27 \mathrm{~mm} / \mathrm{m}^{2}$.

Etiology of PPCM is generally unrecognized; however, there are several presumable causes for PPCM, including viral infection, abnormal reactions in the immunology system, circulatory overload accompanied by pregnancy, and endocrine disorders. Considering the difference of incidence between the US and Japan, etiology of PPCM may chiefly depend on ethnicity. With the growing awareness of this disease, the number of cases diagnosed as PPCM is expected to increase. PPCM is also associated with the occurrence of myocarditis or potential dilated cardiomyopathy caused by cardiac over-
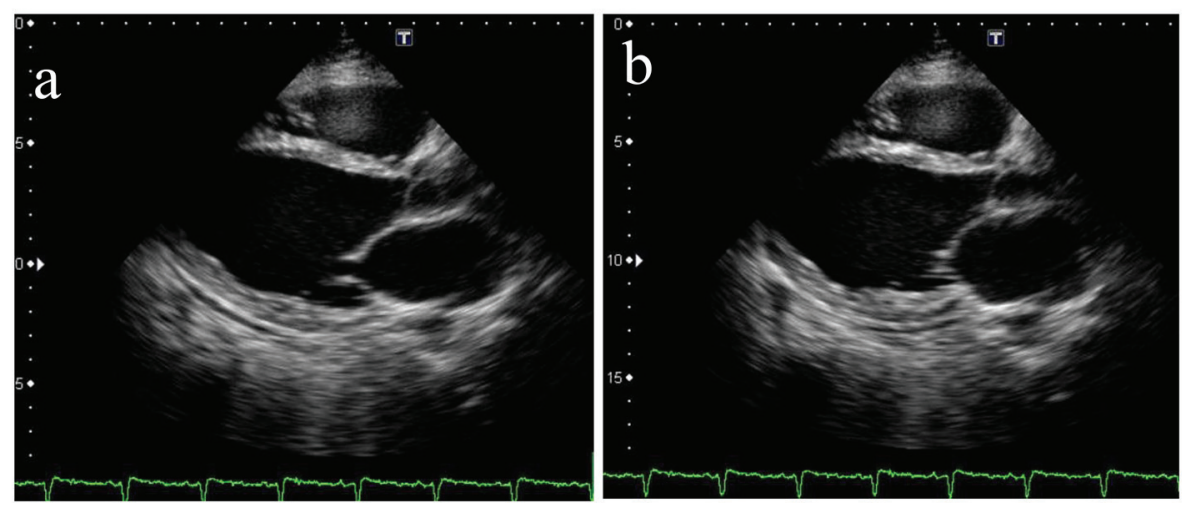

Figure 2. Transthoracic echocardiogram revealed a dilated left ventricle of $63 \mathrm{~mm}$ in diastolic phase (a) and $58 \mathrm{~mm}$ in systolic phase (b) with a reduced EF of $17 \%$. 


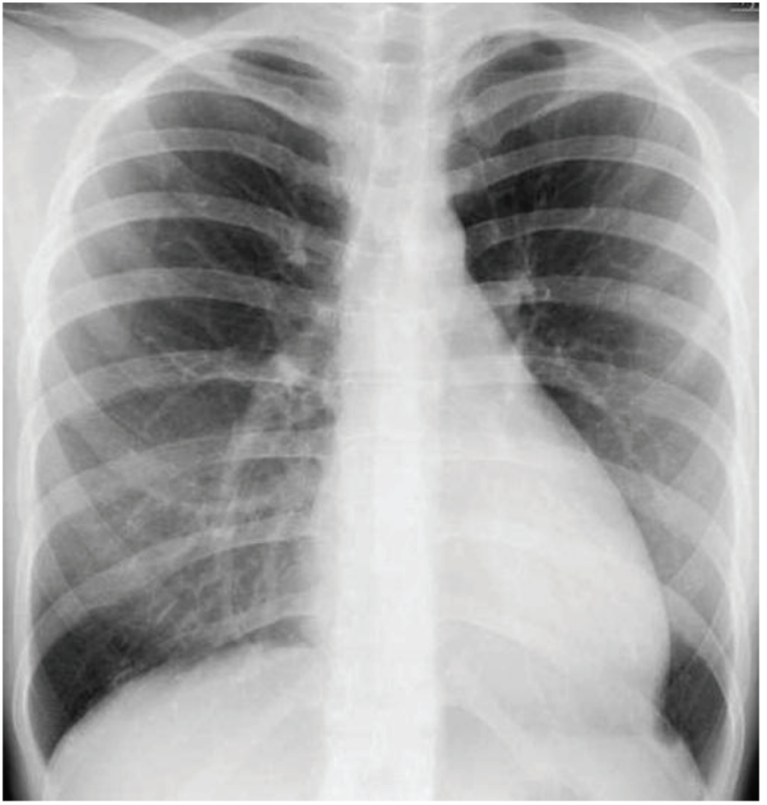

Figure 3. Chest $\mathrm{X}$-ray revealed disappearance of heart congestion and cardiomegaly on day 6 .

load in prenatal and postnatal period. Recent animal experiments focused on the association with prolactin or angiogenic growth factor [4].

The risk factors of PPCM include a multiple pregnancy, advanced age pregnancy, prolific childbirth, people of African descent, gestational hypertension, history of hypertension, smoking, obesity, and use of tocolytic agents [5]. Among these factors, pregnancy hypertension is the biggest risk factor; approximately $57 \%$ of patients with PPCM are reported to have associated pregnancy hypertension, with no difference between races [6]. In 50\% of patients with PPCM, cardiac function is restored, whereas $50 \%$ of patients have reduced cardiac function. In our case, the patient's cardiac function did not improve, as observed on the 6-month follow-up. A small number of patients deteriorate and require heart transplant or risk death.

Treatment for PPCM in its acute phase is a standardized strategy for heart failure, including diuretic drugs, beta blockers, catecholamine, and mechanical circulatory support devices (intra-aortic balloon pumping and percutaneous cardiopulmonary support). In its chronic phase, oral drugs, including angiotensin converting enzyme inhibitors, beta blockers, and diuretic drugs, are generally used. In patients with severe and refractory heart failure, heart transplant is required. In some patients with PPCM, immunosuppressive agents and anti-prolactin drugs are attempted. There is no established consensus or guideline on the cessation of medical treatment in the chronic phase.

Seventeen percent of PPCM cases are reported to have associated ventricle thrombus. This may be caused by the hypercoagulable status during the perinatal period or congestive blood flow with reduced cardiac function [3]. Immediate anti-coagulant therapy is effective in reducing the ventricle thrombus $[7,8]$. It is unclear whether anti-coagulant therapy is necessary in congestive heart failure patients with a normal sinus rhythm because of hemorrhagic risks; anti-coagulant therapy is recommended for congestive heart failure patients in atrial fibrillation [9]. In patients with PPCM, administration of anti-coagulant agents should be considered to prevent thromboembolic complications due to the hypercoagulable status.

In our case, coagulation test was studied on day 10 when the left ventricle thromboses were evident, revealing elevated levels of plasmin- $\alpha 2$ plasmin inhibitor complex $1.9 \mu \mathrm{g} / \mathrm{mL}$ (normal levels $<0.8 \mu \mathrm{g} / \mathrm{mL}$ ) and thrombin-antithrombin complex $3.6 \mu \mathrm{g} / \mathrm{mL}$ (normal levels $<3.0 \mu \mathrm{g} / \mathrm{mL}$ ). This result might suggest that the overexpression of plasminogen activator inhibitor-1. Hence, reduction in thromboses in this patient was expected.

In our patient, upper abdominal pain at admission was due to congestive cholestasis. The mechanism of upper abdominal pain in patients with congestive heart failure is to occur mainly from the expansion of hepatic cells by elevated sinusoidal pressure caused by cholestasis [10]. An additional mechanism of congestive heart failure due to gallbladder edema and infection may cause decreased blood flow in cystic artery, leading to acalculous cholecystitis [10]. Not only with imaging modalities but also with clinical symptoms and manifestation, it was crucial to recognize the presence of congestive heart failure in
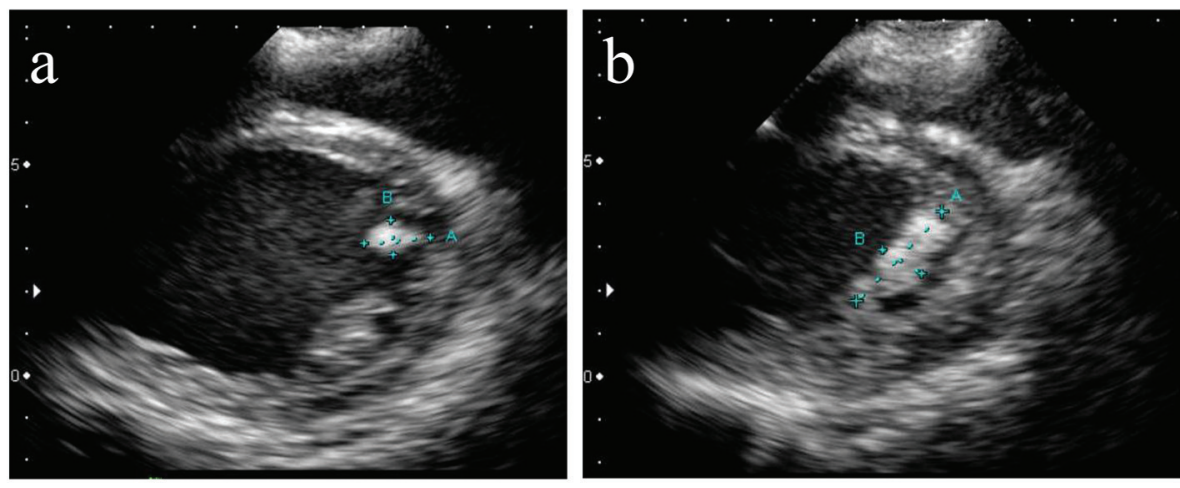

Figure 4. Transthoracic echocardiogram revealed thromboses of $16 \times 8$ (a) $\mathrm{mm}$ and $29 \times 11 \mathrm{~mm}(\mathrm{~b})$ in the patient's left ventricle on day 10. 
this patient.

\section{Conclusion}

PPCM is one of the differential diagnoses in cardiomyopathy that occurs in perinatal or postnatal women. Clinicians should remember that PPCM may be associated with ventricle thrombus caused by the hypercoagulable state in the perinatal period or vascular congestion in reduced cardiac function. They should also remember that PPCM may present gastrointestinal symptoms due to liver congestion.

\section{Conflicts of Interest}

The authors declare that there are no conflicts of interest regarding the publication of this paper.

\section{References}

1. Kolte D, Khera S, Aronow WS, Palaniswamy C, Mujib M, Ahn C, Jain D, et al. Temporal trends in incidence and outcomes of peripartum cardiomyopathy in the United States: a nationwide population-based study. J Am Heart Assoc. 2014;3(3):e001056.

2. Kamiya CA, Kitakaze M, Ishibashi-Ueda H, Nakatani S, Murohara T, Tomoike H, Ikeda T. Different characteristics of peripartum cardiomyopathy between patients com- plicated with and without hypertensive disorders. Results from the Japanese Nationwide survey of peripartum cardiomyopathy. Circ J. 2011;75(8):1975-1981.

3. Demakis JG, Rahimtoola SH. Peripartum cardiomyopathy. Circulation. 1971;44(5):964-968.

4. Hilfiker-Kleiner D, Kaminski K, Podewski E, Bonda T, Schaefer A, Sliwa K, Forster O, et al. A cathepsin Dcleaved $16 \mathrm{kDa}$ form of prolactin mediates postpartum cardiomyopathy. Cell. 2007;128(3):589-600.

5. Amos AM, Jaber WA, Russell SD. Improved outcomes in peripartum cardiomyopathy with contemporary. Am Heart J. 2006;152(3):509-513.

6. Bello N, Rendon IS, Arany Z. The relationship between pre-eclampsia and peripartum cardiomyopathy: a systematic review and meta-analysis. J Am Coll Cardiol. 2013;62(18):1715-1723.

7. Kim DY, Islam S, Mondal NT, Mussell F, Rauchholz M. Biventricular thrombi associated with peripartum cardiomyopathy. J Health Popul Nutr. 2011;29(2):178-180.

8. Nishi I, Ishimitsu T, Ishizu T, Ueno Y, Suzuki A, Seo Y, Ohtsuka S, et al. Peripartum cardiomyopathy and biventricular thrombi. Circ J. 2002;66(9):863-865.

9. Cleland JG, Findlay I, Jafri S, Sutton G, Falk R, Bulpitt C, Prentice C, et al. The Warfarin/Aspirin Study in Heart failure (WASH): a randomized trial comparing antithrombotic strategies for patients with heart failure. Am Heart J. 2004;148(1):157-164.

10. Laurila J, Syrjala H, Laurila PA, Saarnio J, Ala-Kokko TI. Acute acalculous cholecystitis in critically ill patients. Acta Anaesthesiol Scand. 2004;48(8):986-991. 